\title{
ZEROS OF PARTIAL SUMS AND REMAINDERS OF POWER SERIES
}

\author{
BY \\ J. D. BUCKHOLTZ AND J. K. SHAW
}

\begin{abstract}
For a power series $f(z)=\sum_{k=0}^{\infty} a_{k} z^{k}$ let $s_{n}(f)$ denote the maximum modulus of the zeros of the $n$th partial sum of $f$ and let $r_{n}(f)$ denote the smallest modulus of a zero of the $n$th normalized remainder $\sum_{k=n}^{\infty} a_{k} z^{k-n}$. The present paper investigates the relationships between the growth of the analytic function $f$ and the behavior of the sequences $\left\{s_{n}(f)\right\}$ and $\left\{r_{n}(f)\right\}$. The principal growth measure used is that of $R$-type: if $R=\left\{R_{n}\right\}$ is a nondecreasing sequence of positive numbers such that $\lim \left(R_{n+1} / R_{n}\right)=1$, then the $R$-type of $f$ is $\tau_{R}(f)=\lim \sup \left|a_{n} R_{1} R_{2} \cdots R_{n}\right|^{1 / n}$. We prove that there is a constant $P$ such that
\end{abstract}

$$
\tau_{R}(f) \lim \inf \left(s_{n}(f) / R_{n}\right) \leqq P \quad \text { and } \quad \tau_{R}(f) \lim \sup \left(r_{n}(f) / R_{n}\right) \geqq(1 / P)
$$

for functions $f$ of positive finite $R$-type. The constant $P$ cannot be replaced by a smaller number in either inequality; $P$ is called the power series constant.

1. Introduction. The following theorem is a consequence of results of the first author [3] and J. L. Frank [4].

THEOREM A. Let $f(z)=\sum_{k=0}^{\infty} a_{k} z^{k}$ have radius of convergence $c(f), 0<c(f)<\infty$. There exists an absolute constant $P$ such that, if $\varepsilon>0$, then

(i) infinitely many of the partial sums

$$
S_{n}(f ; z)=\sum_{k=0}^{n} a_{k} z^{k} \quad(n=1,2,3, \ldots)
$$

have all their zeros in the disc $|z| \leqq c(f)(P+\varepsilon)$;

(ii) infinitely many of the normalized remainders

$$
\mathscr{S}^{n} f(z)=\sum_{k=n}^{\infty} a_{k} z^{k-n} \quad(n=0,1,2, \ldots)
$$

have no zero in the disc $|z| \leqq c(f)(P+\varepsilon)^{-1}$;

(iii) the constant $P$ cannot be replaced by a smaller number in either (i) or (ii).

In view of (iii), the constant $P$ is uniquely determined by Theorem $\mathrm{A}$. We call $\boldsymbol{P}$ the power series constant; its numerical value is known to lie between 1.7818 and

Presented to the Society, January 23, 1970 under the title Partial sums and remainders of power series; received by the editors May 4, 1971.

AMS 1970 subject classifications. Primary 30A08, 30A10; Secondary 30A06.

Key words and phrases. The power series constant, zeros of partial sums, zeros of remainders, $R$-type, entire functions, extremal functions. 
1.82. Our object in the present paper is to give a simpler proof of Theorem A, to investigate the extremal functions associated with it, and to obtain corresponding results for various classes of entire functions.

For $f(z)=\sum_{k=0}^{\infty} a_{k} z^{k}$, let $s_{n}=s_{n}(f)$ denote the largest of the moduli of the zeros of $S_{n}(f ; z)=\sum_{k=0}^{n} a_{k} z^{k}(n=1,2,3, \ldots)$ with the convention that $s_{n}=\infty$ if $a_{n}=0$. Let $r_{n}=r_{n}(f)$ denote the supremum of numbers $r$ such that $\mathscr{S}^{n} f(z)=\sum_{k=n}^{\infty} a_{k} z^{k-n}$ is analytic and has no zero in the disc $|z|<r$. Theorem $\mathrm{A}$ is equivalent to the estimates

$$
\begin{aligned}
& \liminf _{n \rightarrow \infty} s_{n}(f) \leqq c(f) P, \\
& \limsup _{n \rightarrow \infty} r_{n}(f) \geqq \frac{c(f)}{P},
\end{aligned}
$$

for $0<c(f)<\infty$, together with the assertion that the constant $P$ is best possible in both cases.

Okada [6] has shown that lim $\sup _{n \rightarrow \infty} s_{n}(f)=\infty$ if and only if $f$ is entire. For entire $f$, M. Tsuji [6] proved the surprising result that

$$
\limsup _{n \rightarrow \infty} \frac{\log n}{\log s_{n}(f)}
$$

is always equal to the order of $f$. For functions of positive finite order and type, we are able to sharpen Tsuji's theorem considerably.

THEOREM B. Suppose the entire function $f$ is of order $\rho$ and type $\tau, 0<\rho, \tau<\infty$. Then

$$
\limsup _{n \rightarrow \infty}\left(\frac{\rho \tau}{n}\right)^{1 / \rho} r_{n}(f) \geqq \frac{1}{P}
$$

and

$$
e^{-1 / \rho} \leqq \liminf _{n \rightarrow \infty}\left(\frac{\rho \tau}{n}\right)^{1 / \rho} s_{n}(f) \leqq P .
$$

Furthermore, for each of the three inequalities, there exists an $f$ of order $\rho$ and type $\tau$ for which equality is assumed.

Both Theorem A and Theorem B are special cases of a result involving a more general measure of growth for analytic functions. Let $R=\left\{R_{n}\right\}_{n=1}^{\infty}$ be a nondecreasing sequence of positive numbers such that $\lim _{n \rightarrow \infty} R_{n+1} / R_{n}=1$. The $R$-type, $\tau_{R}(f)$, of an analytic function $f(z)=\sum_{k=0}^{\infty} a_{k} z^{k}$ is defined to be

$$
\tau_{R}(f)=\limsup _{n \rightarrow \infty}\left|a_{n} R_{1} R_{2} \cdots R_{n}\right|^{1 / n} .
$$

If $R_{n} \rightarrow \infty$ as $n \rightarrow \infty, R$-type can be related to the growth of the maximum modulus of $f[1$, p. 6]. It follows easily from the expression for the type of an entire function in terms of its coefficients that $f$ is of order $\rho$ and type $\tau, 0<\rho, \tau<\infty$, if and only if 
$\tau_{R}(f)=1$ for the sequence $R_{n}=(n / \rho \tau)^{1 / \rho}, n=1,2,3, \ldots$ If $\lim _{n \rightarrow \infty} R_{n}=l<\infty$, then one sees that $\tau_{R}(f)=l / c(f)$.

Our principal result is the following.

THEOREM C. If $0<\tau_{R}(f)<\infty$, then

$$
\liminf _{n \rightarrow \infty} \frac{\left(R_{1} R_{2} \cdots R_{n}\right)^{1 / n}}{R_{n}} \leqq \tau_{R}(f) \liminf _{n \rightarrow \infty} \frac{s_{n}(f)}{R_{n}} \leqq P
$$

and

$$
\tau_{R}(f) \limsup _{n \rightarrow \infty} \frac{r_{n}(f)}{R_{n}} \geqq \frac{1}{P}
$$

Furthermore, for each of the three inequalities, there exists a function of $R$-type 1 for which equality is assumed.

If one takes $R_{n} \equiv 1$, Theorem C reduces to Theorem A. If one takes $R_{n} \equiv(n / \rho \tau)^{1 / \rho}$, then Theorem $\mathrm{C}$ reduces to Theorem $\mathrm{B}$.

Suppose $0<c(f)<\infty$ and $\varepsilon>0$. In 1906, M. B. Porter [5] proved that an infinite sequence of the partial sums of $f$ tends uniformly to $\infty$ outside the disc $|z|$ $\leqq c(f)(2+\varepsilon)$. In view of Theorem A, the constant 2 in Porter's theorem cannot be replaced by a number less than $P$. We prove in $\$ 2$ that the best possible constant for Porter's theorem is $P$. This follows fairly easily from a theorem on the partial sums of polynomials which is of some interest in itself.

THEOREM D. Let $Q(z)=a_{0}+a_{1} z+\cdots+a_{n} z^{n}$ be a polynomial of degree $n$. Then for at least one integer $k, 0 \leqq k \leqq n$, we have

$$
\left|a_{0}+a_{1} z+\cdots+a_{k} z^{k}\right| \geqq\left|a_{n}\right||z|^{k} /(n+1)
$$

for all $|z| \geqq P$.

Theorem D guarantees that the partial sum $a_{0}+a_{1} z+\cdots+a_{k} z^{k}$ has all its zeros in the disc $|z| \leqq P$. Since (1.7) holds for large $|z|$, we must have

$$
\left|a_{k}\right| \geqq\left|a_{n}\right| /(n+1) \text {. }
$$

In applications, this yields information about the value of $k$ for which (1.7) holds.

2. The remainder polynomials. The treatment of the power series constant in [3] and [4] involves the remainder polynomials $B_{n}\left(z ; z_{0}, z_{1}, \ldots, z_{n-1}\right)$, defined recursively by

$$
\begin{aligned}
B_{0}(z) & =1, \\
B_{n}\left(z ; z_{0}, z_{1}, \ldots, z_{n-1}\right) & =z^{n}-\sum_{k=0}^{n-1} z_{k}^{n-k} B_{k}\left(z ; z_{0}, z_{1}, \ldots, z_{k-1}\right) .
\end{aligned}
$$

Let

$$
H_{n}=\max \left|B_{n}\left(0 ; z_{0}, \ldots, z_{n-1}\right)\right|
$$


where the maximum is taken over all sequences $\left\{z_{k}\right\}_{k=0}^{n-1}$ whose terms lie on $|z|=1$. Buckholtz [3] proved that

$$
P=\lim _{n \rightarrow \infty} H_{n}^{1 / n}=\sup _{1 \leqq n<\infty} H_{n}^{1 / n} .
$$

For a power series $f(z)=\sum_{k=0}^{\infty} a_{k} z^{k}$, we write (2.1) in the form

$$
z^{n}=\sum_{k=0}^{n} z_{k}^{n-k} B_{k}\left(z ; z_{0}, \ldots, z_{k-1}\right)
$$

and substitute this expression into the power series for $f$. We obtain the formal expansion

$$
\begin{aligned}
f(z) & =\sum_{n=0}^{\infty} a_{n} z^{n}=\sum_{n=0}^{\infty} a_{n}\left\{\sum_{k=0}^{n} z_{k}^{n-k} B_{k}\left(z ; z_{0}, \ldots, z_{k-1}\right)\right\} \\
& =\sum_{k=0}^{\infty} B_{k}\left(z ; z_{0}, \ldots, z_{k-1}\right) \sum_{n=k}^{\infty} a_{n} z_{k}^{n-k}=\sum_{k=0}^{\infty} \mathscr{S}^{k} f\left(z_{k}\right) B_{k}\left(z ; z_{0}, \ldots, z_{k-1}\right),
\end{aligned}
$$

which holds whenever the interchange in the order of summation can be justified. In particular, (2.2) holds if $f$ is a polynomial and yields considerable information when $f$ is taken to be a remainder polynomial. In the latter case, an easy induction argument establishes the identity

$$
\mathscr{S}^{k} B_{n}\left(z ; z_{0}, \ldots, z_{n-1}\right)=B_{n-k}\left(z ; z_{k}, \ldots, z_{n-1}\right),
$$

for $0 \leqq k \leqq n$.

The remainder polynomials also satisfy the following properties:

(2.4) $B_{n}\left(\lambda z ; \lambda z_{0}, \ldots, \lambda z_{n-1}\right)=\lambda^{n} B_{n}\left(z ; z_{0}, \ldots, z_{n-1}\right)$,

(2.5) $B_{n}\left(z_{0} ; z_{0}, \ldots, z_{n-1}\right)=0$,

(2.6) $z^{n} B_{n}\left(1 / z ; z_{n}, \ldots, z_{1}\right)=\sum_{k=0}^{n} B_{k}\left(0 ; z_{k}, \ldots, z_{1}\right) z^{k}$,

(2.7) $B_{n}\left(z ; z_{n}, \ldots, z_{1}\right)=\sum_{k=0}^{n_{1}} B_{k}\left(0 ; z_{k}, \ldots, z_{1}\right) B_{n-k}\left(z ; z_{n}, \ldots, z_{n_{1}+1}, 0, \ldots, 0\right)$ for $0 \leqq n_{1} \leqq n$,

(2.8) $H_{m+n} \geqq H_{m} H_{n}$ for nonnegative integers $m$ and $n$.

The proofs of these identities may be found in [3].

We are now ready to prove Theorem $\mathrm{D}$. Thus let $Q(z)=a_{0}+a_{1} z+\cdots+a_{n} z^{n}$ be a polynomial of degree $n$. Define $f(z)=z^{n} Q(1 / z)=b_{0}+b_{1} z+\cdots+b_{n} z^{n}$; note that $b_{n-k}=a_{k}, 0 \leqq k \leqq n$. Let $\left\{z_{j}\right\}_{j=0}^{n}$ be a sequence of complex numbers satisfying

$$
\left|\mathscr{S}^{j} f\left(z_{j}\right)\right|=\min _{|z| \leqq 1 / P}\left|\mathscr{S}^{j} f(z)\right|, \quad 0 \leqq j \leqq n .
$$

From (2.2),

$$
|f(0)| \leqq \sum_{k=0}^{n}\left|\mathscr{S}^{k} f\left(z_{k}\right)\right|\left|B_{k}\left(0 ; z_{0}, \ldots, z_{k-1}\right)\right| .
$$

Setting $w_{k}=P z_{k}, 0 \leqq k \leqq n$, we have $\left|w_{k}\right| \leqq 1$ and, by (2.4),

$$
\begin{aligned}
\left|B_{k}\left(0 ; z_{0}, \ldots, z_{k-1}\right)\right| & =\left|B_{k}\left(0 ; w_{0} / P, \ldots, w_{k-1} / P\right)\right| \\
& =\left(1 / P^{k}\right)\left|B_{k}\left(0 ; w_{0}, \ldots, w_{k-1}\right)\right| \leqq\left(1 / P^{k}\right) H_{k} \leqq 1,
\end{aligned}
$$


for $0 \leqq k \leqq n$. Hence $|f(0)| \leqq \sum_{k=0}^{n}\left|\mathscr{S}^{k} f\left(z_{k}\right)\right|$ and so $|f(0)| \leqq(n+1)\left|\mathscr{S}^{m} f\left(z_{m}\right)\right|$ for some $m, 0 \leqq m \leqq n$. Since $f(0)=b_{0}$, we have $\left|\mathscr{S}^{m} f(z)\right| \geqq\left|b_{0}\right| /(n+1)$ for all $|z| \leqq 1 / P$. Now

$$
\mathscr{S}^{m} f(z)=b_{m}+b_{m+1} z+\cdots+b_{n} z^{n-m}
$$

and therefore, replacing $z$ by $1 / z$, we obtain

$$
\left|b_{m} z^{n-m}+b_{m+1} z^{n-m-1}+\cdots+b_{n}\right| \geqq|z|^{n-m}\left|b_{0}\right| /(n+1)
$$

for all $|z| \geqq P$. Letting $p=n-m$, this inequality is equivalent to

$$
\left|a_{0}+a_{1} z+\cdots+a_{p} z^{p}\right| \geqq|z|^{p}\left|a_{n}\right| /(n+1)
$$

for all $|z| \geqq P$, and this completes the proof.

COROLlaRY 1. Suppose that the power series $\sum_{k=0}^{\infty} a_{k} z^{k}$ has radius of convergence less than 1. Then there are infinitely many integers $k$ such that

$$
\left|\sum_{j=0}^{k} a_{j} z^{j}\right| \geqq|z|^{k}
$$

for all $|z| \geqq P$.

Proof. For each positive integer $n$ such that $a_{n} \neq 0$, let $k(n)$ denote the least positive integer $k$ for which (1.7) holds. The condition lim sup $\left|a_{n}\right|^{1 / n}>1$ implies that there is an infinite set $I$ of positive integers such that $\left|a_{n}\right| /(n+1)>n$ for all $n \in I$. For each $n \in I$ we therefore have $\left|\sum_{j=0}^{k(n)} a_{j} z^{j}\right| \geqq|z|^{k(n)}$ and, by (1.8), $\left|a_{k(n)}\right|$ $\geqq\left|a_{n}\right| /(n+1)>n$. The latter condition guarantees that $k(n)$ assumes infinitely many values as $n$ ranges over $I$, and this completes the proof.

Suppose $f$ has radius of convergence $t, 0<t<\infty$. Let $\varepsilon>0$ and define $g(z)$ $=f(t z /(1-\varepsilon))$. Then $c(g)<1$ and $(2.9)$ implies that $s_{n}(g) \leqq P$ for infinitely many integers $n$. Thus $\liminf _{n \rightarrow \infty} s_{n}(g) \leqq P$. But $s_{n}(g)=((1-\varepsilon) / t) s_{n}(f)$ and therefore $\lim \inf _{n \rightarrow \infty} s_{n}(f) \leqq t P /(1-\varepsilon)$. It follows that $\lim \inf _{n \rightarrow \infty} s_{n}(f) \leqq c(f) P$ and this proves (1.1).

LEMMA 1. If $n$ is a nonnegative integer, then

$$
1 \leqq P^{n} / H_{n} \leqq 17 \text {. }
$$

This will be proved in $\S 3$.

Let $m$ be a positive integer and suppose $z_{0}, z_{1}, \ldots, z_{m-1}$ lie on $|z|=1$. If $k \geqq m$, then (2.1) implies

$$
B_{k}\left(0 ; z_{0}, \ldots, z_{m-1}, 0, \ldots, 0\right)=-\sum_{j=0}^{m-1} z_{j}^{k-j} B_{j}\left(0 ; z_{0}, \ldots, z_{j-1}\right) .
$$

It follows that

$$
\left|B_{k}\left(0 ; z_{0}, \ldots, z_{m-1}, 0, \ldots, 0\right)\right| \leqq \sum_{j=0}^{m-1} H_{j} \leqq \sum_{j=0}^{m-1} P^{j}<\frac{P^{m}}{P-1} .
$$


The assertion that the constant $P$ is best possible in (1.1) depends on the existence of a function $f$ such that $\lim \inf s_{n}(f)=c(f) P$. It suffices to construct such an $f$ satisfying $c(f)=1$.

Lemma 2. There exists a power series $\sum_{k=0}^{\infty} A_{k} z^{k}$, with radius of convergence 1 , such that each partial sum $\sum_{k=0}^{m} A_{k} z^{k}$ has a zero of modulus $P$.

Proof. For each nonnegative integer $n$, let $\left\{z_{j}^{(n)}\right\}_{j=1}^{n}$ be a sequence of complex numbers of modulus $1 / P$ such that

$$
\left|B_{n}\left(0 ; z_{n}^{(n)}, z_{n-1}^{(n)}, \ldots, z_{1}^{(n)}\right)\right|=H_{n} / P^{n} .
$$

Here, we have used (2.4). If $n, n_{1}$ and $j$ are positive integers such that $j \leqq n_{1} \leqq n$, then (2.7) implies

$$
\begin{aligned}
& \left|B_{n}\left(0 ; z_{n}^{(n)}, \ldots, z_{1}^{(n)}\right)\right| \\
& \quad \leqq \\
& \quad \sum_{k=0}^{n_{1}-j} \mid B_{k}\left(0 ; z_{k}^{(n)}, \ldots, z_{1}^{(n)}|| B_{n-k}\left(0 ; z_{n}^{(n)}, \ldots, z_{n_{1}+1}^{(n)}, 0, \ldots, 0\right) \mid\right. \\
& \quad+\sum_{k=n_{1}-j+1}^{n_{1}}\left|B_{k}\left(0 ; z_{k}^{(n)}, \ldots, z_{1}^{(n)}\right)\right|\left|B_{n-k}\left(0 ; z_{n}^{(n)}, \ldots, z_{n_{1}+1}^{(n)}, 0, \ldots, 0\right)\right| .
\end{aligned}
$$

If $0 \leqq k \leqq n_{1}-j$, we have

$$
\mid B_{k}\left(0 ; z_{k}^{(n)}, \ldots, z_{1}^{(n)} \mid \leqq H_{k} / P^{k} \leqq 1\right.
$$

and (2.11) implies

$$
\left|B_{n-k}\left(0 ; z_{n}^{(n)}, \ldots, z_{n_{1}+1}^{(n)}, 0, \ldots, 0\right)\right| \leqq P^{n-n_{1}} /\left(P^{n-k}(P-1)\right) .
$$

The first sum on the right of (2.13) therefore does not exceed

$$
\sum_{k=0}^{n_{1}-j} \frac{P^{k-n_{1}}}{(P-1)}=\frac{P^{-j+1}-P^{-n_{1}}}{(P-1)^{2}}<\frac{1}{P^{j-1}(P-1)^{2}} .
$$

If $n_{1}-j+1 \leqq k \leqq n_{1}$, then

$$
\left|B_{n-k}\left(0 ; z_{n}^{(n)}, \ldots, z_{n_{1}+1}^{(n)}, 0, \ldots, 0\right)\right| \leqq H_{n-k} / P^{n-k} \leqq 1 .
$$

In view of (2.10), (2.13) now yields

$$
\sum_{k=n_{1}-j+1}^{n_{1}}\left|B_{k}\left(0 ; z_{k}^{(n)}, \ldots, z_{1}^{(n)}\right)\right| \geqq \frac{1}{17}-\frac{1}{P^{j-1}(P-1)^{2}} .
$$

Taking $j=7$ and using the bound $P>1.78$, we have $\sum_{k=n_{1}-6}^{n_{1}}\left|B_{k}\left(0 ; z_{k}^{(n)}, \ldots, z_{1}^{(n)}\right)\right|$ $>1 / 1000$. Therefore $\left|B_{k}\left(0 ; z_{k}^{(n)}, \ldots, z_{1}^{(n)}\right)\right|>1 / 7000$ for at least one integer $k, n_{1}-6$ $\leqq k \leqq n_{1}$. Moreover, $\left|B_{k}\left(0 ; z_{k}^{(n)}, \ldots, z_{1}^{(n)}\right)\right| \leqq 1$ for all $n$ and $k$. Now define

$$
P_{n}(z)=z^{n} B_{n}\left(1 / z ; z_{n}^{(n)}, \ldots, z_{1}^{(n)}\right), \quad n=0,1,2, \ldots
$$

Since (2.6) implies $P_{n}(z)=\sum_{k=0}^{n} B_{k}\left(0 ; z_{k}^{(n)}, \ldots, z_{1}^{(n)}\right) z^{k}$, it follows that the coefficients of $P_{n}$ are bounded by 1 and that in a set of 7 consecutive coefficients, at least one 
coefficient has modulus greater than $1 / 7000$. The sequence $\left\{P_{n}\right\}$ is uniformly bounded on compact subsets of the unit disc. Extract a uniformly convergent subsequence of $\left\{P_{n}\right\}$ and let $F$ denote the limit function. Writing $F(z)=\sum_{k=0}^{\infty} A_{k} z^{k}$, it follows that $\left|A_{k}\right| \leqq 1,0 \leqq k<\infty$, and that in a set of 7 consecutive coefficients $A_{k}$, at least one coefficient has modulus greater than $1 / 7000$. Hence $c(F)=1$. If $m<n$, then (2.6) implies that the $m$ th partial sum of $P_{n}$ is given by

$$
S_{m}\left(P_{n} ; z\right)=z^{m} B_{m}\left(1 / z ; z_{m}^{(n)}, \ldots, z_{1}^{(n)}\right) .
$$

By (2.5), $S_{m}\left(P_{n} ; 1 / z_{m}^{(n)}\right)=0$. Since $S_{m}(F ; z)$ is the uniform limit of a subsequence of $\left\{S_{m}\left(P_{n} ; z\right)\right\}$, it follows that $S_{m}(F ; z)$ has a zero of modulus $P$. This completes the proof of the lemma.

The function $F$ of the preceding lemma satisfies $c(F)=1$ and $\lim _{\inf _{n \rightarrow \infty}} s_{n}(F) \geqq P$. It follows that the constant $P$ is best possible in (1.1).

We now show that $P$ is the sharp constant in Porter's theorem. If $f(z)$ $=\sum_{k=0}^{\infty} a_{k} z^{k}$ has radius of convergence $t$, then Corollary 1 implies that there are infinitely many integers $k$ such that $\left|\sum_{j=0}^{k} \dot{a}_{j} z^{j}\right| \geqq(|z| / t(1+\varepsilon))^{k}$ for all $|z| \geqq t P(1+\varepsilon)$. The corresponding subsequence of partial sums $\left\{S_{k}(f ; z)\right\}$ therefore tends uniformly to $\infty$ outside the $\operatorname{disc}|z| \leqq c(f) P(1+\varepsilon)$. On the other hand, we can, by Lemma 3, construct a function $F$ such that $c(F)=t$ and such that each partial sum of $F$ has a zero in $|z| \leqq c(F) P$.

The inequality (1.2) is a special case of (1.6); the latter will be proved in $\$ 4$. To show that $P$ is the sharp constant in (1.2), it suffices to construct a function $G$ satisfying $c(G)=1$ and $\lim \sup r_{n}(G) \leqq 1 / P$.

Lemma 3. There exists a power series $G(z)=\sum_{k=0}^{\infty} A_{k} z^{k}$, with $c(G)=1$, such that each normalized remainder of $G$ has a zero of modulus $1 / P$. In particular, lim $\sup r_{n}(G)$ $\leqq 1 / P$.

Proof. Consider the sequence of complex numbers $\left\{B_{n}\left(0 ; z_{n}^{(n)}, \ldots, z_{1}^{(n)}\right)\right\}_{n=1}^{\infty}$ constructed in Lemma 2. For each $n$ we have $\left|z_{j}^{(n)}\right|=1 / P$, for $1 \leqq j \leqq n$, $\left|B_{j}\left(0 ; z_{j}^{(n)}, \ldots, z_{1}^{(n)}\right)\right| \leqq 1$, for $0 \leqq j \leqq n$, and $\left|B_{n}\left(0 ; z_{n}^{(n)}, \ldots, z_{1}^{(n)}\right)\right|=H_{n} / P^{n}$. Furthermore, if $n_{1} \leqq n$, then $\left|B_{k}\left(0 ; z_{k}^{(n)}, \ldots, z_{1}^{(n)}\right)\right| \geqq 1 / 7000$ for at least one integer $k$ such that $n_{1}-6 \leqq k \leqq n_{1}$. By (2.6),

$$
B_{n}\left(z ; z_{n}^{(n)}, \ldots, z_{1}^{(n)}\right)=\sum_{k=0}^{n} B_{k}\left(0 ; z_{k}^{(n)}, \ldots, z_{1}^{(n)}\right) z^{n-k} .
$$

The sequence $\left\{B_{n}\left(z ; z_{n}^{(n)}, \ldots, z_{1}^{(n)}\right)\right\}_{n=1}^{\infty}$ is therefore uniformly bounded on compact subsets of the unit disc. Extract a uniformly convergent subsequence from $\left\{B_{n}\right\}$ and let $G$ denote the limit function. If $G(z)=\sum_{k=0}^{\infty} A_{k} z^{k}$, then $\left|A_{k}\right| \leqq 1$ for all $k$ and $\left|A_{k}\right| \geqq 1 / 7000$ for infinitely many $k$; thus $c(G)=1$. The identities

$$
\begin{gathered}
\mathscr{S} k^{k} B_{n}\left(z ; z_{n}^{(n)}, \ldots, z_{1}^{(n)}\right)=B_{n-k}\left(z ; z_{n-k}^{(n)}, \ldots, z_{1}^{(n)}\right), \\
B_{n-k}\left(z_{n-k}^{(n)} ; z_{n-k}^{(n)}, \ldots, z_{1}^{(n)}\right)=0,
\end{gathered}
$$


for $0 \leqq k<n$, show that $B_{n}$ and each of its first $(n-1)$ normalized remainders have zeros of modulus $1 / P$. Furthermore, if $m$ is a nonnegative integer, then $\mathscr{S}^{m} G(z)$ is the uniform limit of a subsequence of $\left\{\mathscr{S}^{m} B_{n}\left(z ; z_{n}^{(n)}, \ldots, z_{1}^{(n)}\right)\right\}$ on the compact set $|z| \leqq(1 / P)+\varepsilon<1$. It follows that $\mathscr{S}^{m} G(z)$ has a zero of modulus $1 / P$.

3. The functions $T_{m}(\mathscr{U})$. For $m=1,2,3, \ldots$, and $0 \leqq \mathscr{U}<1$, define

$$
T_{m}(\mathscr{U})=\max \sum_{k=m}^{\infty} \mathscr{U}^{k}\left|B_{k}\left(0 ; w_{0}, w_{1}, \ldots, w_{m-1}, 0, \ldots, 0\right)\right|
$$

where the maximum is taken over all sequences $\left\{w_{k}\right\}_{k=0}^{m-1}$ whose terms lie on $|z|=1$. The functions $T_{n}(\mathscr{U})$ were characterized by Buckholtz [3]. For each $m, T_{m}$ is increasing; the unique solution to the equation $T_{m}(\mathscr{U})=1$ is denoted by $\mathscr{U}_{m}$. The most important property of the sequence $\left\{\mathscr{U}_{m}\right\}$ is the determination

$$
\mathbf{P}=\lim _{m \rightarrow \infty} \mathscr{U}_{m}^{-1}=\inf _{1 \leqq m \leqq \infty} \mathscr{U}_{m}^{-1} \text {. }
$$

Since $T_{m}$ is increasing, (3.1) implies

$$
T_{m}(1 / P)>1, \quad m=1,2,3, \ldots
$$

Proof of Lemma 1. By (2.11) and (3.2), we have

$$
1 \leqq T_{m}(1 / P) \leqq \frac{H_{m}}{P^{m}}+\frac{H_{m+1}}{P^{m+1}}+\frac{H_{m+2}}{P^{m+2}}+\sum_{k=m+3}^{\infty}(1 / P)^{k} \frac{P^{m}}{P-1},
$$

for each positive integer $m$.

In view of (2.8), the previous inequality implies

$$
1 \leqq\left(\frac{H_{m+2}}{P^{m+2}}\right)\left[1+\frac{P}{H_{1}}+\frac{P^{2}}{H_{2}}\right]+\frac{P^{m}}{P-1} \frac{P^{-m-3}}{(1-(1 / P))} ;
$$

therefore,

$$
1 \leqq\left(H_{m+2} / P^{m+2}\right)\left[1+P+P^{2} / 2\right]+P^{-2}(P-1)^{-2} .
$$

Using the bounds $1.78<P<1.82$, we obtain $H_{m+2} / P^{m+2} \geqq 1 / 17$. It is easily verified that $H_{j} / P^{j}>1 / 17$ for $j=1,2$. Since $P=\sup _{1 \leqq n<\infty} H_{n}^{1 / n}$, we have $1 / 17 \leqq H_{n} / P^{n} \leqq 1$ for all $n$.

4. Main results. In this section, we prove (1.5) and (1.6).

LEMMA 4. Let $m$ be a positive integer and $\left\{A_{k}\right\}_{k=1}^{\infty}$ a sequence of complex numbers $\left(A_{0}=1\right)$ such that $\left|A_{k}\right| \leqq 1$ for $k \geqq m$. Then for at least one integer $p, 0 \leqq p \leqq m-1$, the function $A_{p}+A_{p+1} z+A_{p+2} z^{2}+\cdots$ has no zero in the disc $|z|<\mathscr{U}_{m}$.

Proof. Let $f(z)=1+\sum_{k=1}^{\infty} A_{k} z^{k}$. We have to show that for some $p, 0 \leqq p \leqq m-1$, $\mathscr{S}^{p} f(z)$ has no zero in $|z|<\mathscr{U}_{m}$. Let $\left\{z_{k}\right\}_{k=0}^{\infty}$ be a sequence of points in $|z|<1$ such that $z_{k}=0$ for $k \geqq m$. Then, by (2.1), 


$$
\begin{array}{rl}
\sum_{k=0}^{m-1} \mathscr{S}^{k} & f\left(z_{k}\right) B_{k}\left(z ; z_{0}, \ldots, z_{k-1}\right) \\
& =\sum_{j=0}^{m-1} A_{j} \sum_{k=0}^{j} z_{k}^{j-k} B_{k}\left(z ; z_{0}, \ldots, z_{k-1}\right)+\sum_{j=m}^{\infty} A_{j} \sum_{k=0}^{m-1} z_{k}^{j-k} B_{k}\left(z ; z_{0}, \ldots, z_{k-1}\right) \\
& =\sum_{j=0}^{m-1} A_{j} z^{j}+\sum_{j=m}^{\infty} A_{j}\left[z^{j}-B_{j}\left(z ; z_{0}, \ldots, z_{m-1}, 0, \ldots, 0\right)\right] \\
& =\sum_{j=0}^{\infty} A_{j} z^{j}-\sum_{j=m}^{\infty} A_{j} B_{j}\left(z ; z_{0}, \ldots, z_{m-1}, 0, \ldots, 0\right) .
\end{array}
$$

By transposing, we obtain the important identity

$$
f(z)=\sum_{k=0}^{m-1} \mathscr{S}^{k} f\left(z_{k}\right) B_{k}\left(z ; z_{0}, \ldots, z_{k-1}\right)+\sum_{k=m}^{\infty} A_{k} B_{k}\left(z ; z_{0}, \ldots, z_{m-1}, 0, \ldots, 0\right) .
$$

Without loss of generality, we may assume that each of $\mathscr{S}^{k} f(z), 0 \leqq k \leqq m-1$, has a zero in $|z|<1$. For $0 \leqq k \leqq m-1$, let $w_{k}$ denote the smallest modulus of a zero of $\mathscr{S}^{k} f(z)$. It follows from (4.1) that

$$
1=f(0) \leqq \sum_{k=m}^{\infty}\left|B_{k}\left(0 ; w_{0}, \ldots, w_{m-1}, 0, \ldots, 0\right)\right| .
$$

If $\mathscr{U}=\max _{0 \leqq k \leqq m}\left|w_{k}\right|$, then

$$
1 \leqq \sum_{k=m}^{\infty} \mathscr{U}^{k}\left|B_{k}\left(0 ; w_{0}\left|\mathscr{U}, \ldots, w_{m-1}\right| \mathscr{U}, 0, \ldots, 0\right)\right| \leqq T_{m}(\mathscr{U})
$$

and therefore $\mathscr{U} \geqq \mathscr{U}_{m}$. Thus there is an integer $p, 0 \leqq p \leqq m-1$, such that $\left|w_{p}\right| \geqq \mathscr{U}_{m}$ and it follows that $\mathscr{S}^{p} f(z)$ has no zero in $|z|<\mathscr{U}_{m}$.

LEMMA 5. Let $m$ be a positive integer and $a_{0}+a_{1} z+\cdots+a_{n} z^{n}$ a polynomial of degree $n, n \geqq m-1$, such that $\left|a_{k}\right| \leqq\left|a_{n}\right|, 0 \leqq k \leqq n$. Then for at least one integer $p$, $n-m+1 \leqq p \leqq n$, the polynomial $a_{0}+a_{1} z+\cdots+a_{p} z^{p}$ has all its zeros in the disc $|z| \leqq \mathscr{U}_{m}^{-1}$.

Proof. Let $A_{k}=a_{n-k} / a_{n}, 0 \leqq k \leqq n$. Lemma 4 implies that there exists an integer $q$, $0 \leqq q \leqq m-1$, such that $A_{q}+A_{q+1} z+\cdots+A_{n} z^{n-q}$ does not vanish in $|z|<\mathscr{U}_{m}$. Therefore, the function $\left(a_{n-q} / a_{n}\right)+\left(a_{n-q-1} / a_{n}\right) z+\cdots+\left(a_{0} / a_{n}\right) z^{n-q}$ has no zero in $|z|<\mathscr{U}_{m}$, so the same is true of $\left(z^{n-q} / a_{n}\right)\left(a_{0}+a_{1} / z+\cdots+a_{n-q} / z^{n-q}\right)$. It follows that $\left(1 / a_{n} z^{n-q}\right)\left(a_{0}+a_{1} z+\cdots+a_{n-q} z^{n-q}\right)$ has no zero in the region $|z|>\mathscr{U}_{m}^{-1}$, hence $a_{0}+a_{1} z+\cdots+a_{n-q} z^{n-q}$ has all its zeros in $|z| \leqq \mathscr{U}_{m}^{-1}$. Taking $p=n-q$, we obtain the desired result.

Lemma 6. Suppose $f(z)=\sum_{k=0}^{\infty} A_{k} z^{k}$ has $R$-type greater than 1. Then

$$
\liminf _{n \rightarrow \infty} \frac{s_{n}(f)}{R_{n}} \leqq P
$$


Proof. If $f(z)$ is written

$$
f(z)=\sum_{k=0}^{\infty}\left(a_{k} / R_{1} R_{2} \cdots R_{k}\right) z^{k}
$$

then $\tau_{R}(f)=\lim \sup _{n \rightarrow \infty}\left|a_{n}\right|^{1 / n}$. The condition $\tau_{R}(f)>1$ implies that there exists an infinite set $N$ of positive integers such that $n \in N$ implies $\left|a_{n}\right|>\left|a_{k}\right|, 0 \leqq k<n$. Let $m$ be a positive integer and suppose $n \in N$ is such that $n \geqq m-1$. The $n$th partial sum of $f\left(R_{n} z\right)$ is given by

$$
\begin{aligned}
& S_{n}\left(f ; R_{n} z\right)=a_{0}+\frac{a_{1} R_{n}}{R_{1}} z+\frac{a_{2} R_{n}^{2}}{R_{1} R_{2}} z^{2}+\cdots+\frac{a_{n} R_{n}^{n}}{R_{1} R_{2} \cdots R_{n}} z^{n} \\
& \quad=\frac{a_{n} R_{n}^{n}}{R_{1} R_{2} \cdots R_{n}}\left(z^{n}+\frac{a_{n-1} R_{n}}{a_{n} R_{n}} z^{n-1}+\frac{a_{n-2} R_{n-1} R_{n}}{a_{n} R_{n}^{2}} z^{n-2}+\cdots+\frac{a_{0} R_{1} R_{2} \cdots R_{n}}{a_{n} R_{n}^{n}}\right) .
\end{aligned}
$$

For $n \in N$ and $n \geqq m-1$, Lemma 5 , applied to the polynomial

$$
z^{n}+\frac{a_{n-1} R_{n}}{a_{n} R_{n}} z^{n-1}+\frac{a_{n-2} R_{n-1} R_{n}}{a_{n} R_{n}^{2}} z^{n-2}+\cdots+\frac{a_{0} R_{1} R_{2} \cdots R_{n}}{a_{n} R_{n}^{n}},
$$

implies that at least one of the partial sums $S_{n}\left(f ; R_{n} z\right), S_{n-1}\left(f ; R_{n} z\right), \ldots$, $S_{n-m+1}\left(f ; R_{n} z\right)$ has all its zeros in the disc $|z| \leqq \mathscr{U}_{m}^{-1}$. In view of $s_{k}\left(f\left(R_{n} z\right)\right)$ $=R_{n}^{-1} s_{k}(f)$, for $n-m+1 \leqq k \leqq n$, it follows that

$$
\min \left\{s_{n}(f) / R_{n}, s_{n-1}(f) / R_{n}, \ldots, s_{n-m+1}(f) / R_{n}\right\} \leqq \mathscr{U}_{m}^{-1}
$$

for all $n \in N, n \geqq m-1$. If $n-k(n)$ denotes the subscript for which the minimum in (4.2) is assumed, then

$$
\left(s_{n-k(n)}(f) / R_{n-k(n)}\right)\left(R_{n-m+1} / R_{n}\right) \leqq \mathscr{U}_{m}^{-1}
$$

for $n \in N, n \geqq m-1$. Since $\lim _{n \rightarrow \infty}\left(R_{n-m+1} / R_{n}\right)=1$, then (4.3) implies

$$
\liminf _{j \rightarrow \infty} \frac{s_{j}(f)}{R_{j}} \leqq \mathscr{U}_{m}^{-1}
$$

Since $m$ is arbitrary, (3.1) implies $\lim \inf _{j \rightarrow \infty} s_{j}(f) / R_{j} \leqq P$, which is the desired result.

For a power series $f(z)=1+\sum_{k=1}^{\infty} a_{k} z^{k}$, the estimate

$$
s_{n}(f) \geqq\left|a_{n}\right|^{-1 / n} \quad\left(a_{n} \neq 0\right)
$$

follows from the fact that the geometric mean of the moduli of the zeros of $S_{n}(f ; z)$ does not exceed the maximum modulus of its zeros. The following lemma, whose proof we omit, is an extension of (4.4).

LEMMA 7. Suppose the power series $f(z)=\sum_{k=0}^{\infty} a_{k} z^{k}$ has positive radius of convergence and is not a polynomial. If $N=\left\{n: a_{n} \neq 0\right\}$, then

$$
\liminf _{n \rightarrow \infty}\left|a_{n}\right|^{1 / n} s_{n}(f) \geqq 1 \text {. }
$$

We are now ready to prove (1.5) of Theorem C. 
THEOREM 1. If $0<\tau_{R}(f)<\infty$, then

$$
\liminf _{n \rightarrow \infty} \frac{\left(R_{1} R_{2} \cdots R_{n}\right)^{1 / n}}{R_{n}} \leqq \tau_{R}(f) \liminf _{n \rightarrow \infty} \frac{s_{n}(f)}{R_{n}} \leqq P .
$$

Proof. If $f(z)=\sum_{k=0}^{\infty} A_{k} z^{k}=\sum_{k=0}^{\infty}\left(a_{k} / R_{1} R_{2} \cdots R_{k}\right) z^{k}$, then

$$
\begin{aligned}
\tau_{R}(f) & =\limsup _{n \rightarrow \infty}\left|a_{n}\right|^{1 / n}=\limsup _{n \rightarrow \infty}\left|A_{n}\right|^{1 / n}\left(R_{1} \cdots R_{n}\right)^{1 / n} \\
& \geqq R_{1} \limsup _{n \rightarrow \infty}\left|A_{n}\right|^{1 / n}=R_{1} / c(f)
\end{aligned}
$$

and therefore $c(f)>0$. Since $\tau_{R}(f)>0, f$ is not a polynomial. By Lemma 7,

$$
\lim _{n \rightarrow \infty} \inf _{n \in N}\left|A_{n}\right|^{1 / n} S_{n}(f) \geqq 1,
$$

where $N=\left\{n: A_{n} \neq 0\right\}$. Therefore,

$$
\begin{aligned}
\liminf _{n \rightarrow \infty} & \frac{\left(R_{1} R_{2} \cdots R_{n}\right)^{1 / n}}{R_{n}} \leqq \liminf _{n \rightarrow \infty: n \in N} \frac{\left(R_{1} R_{2} \cdots R_{n}\right)^{1 / n}}{R_{n}} \liminf _{n \rightarrow \infty ; n \in N}\left|A_{n}\right|^{1 / n} s_{n}(f) \\
& \leqq \liminf _{n \rightarrow \infty ; n \in N} \frac{\left(R_{1} R_{2} \cdots R_{n}\right)^{1 / n}}{R_{n}}\left|A_{n}\right|^{1 / n} S_{n}(f) \\
& \leqq \limsup _{n \rightarrow \infty}\left(R_{1} R_{2} \cdots R_{n}\right)^{1 / n}\left|A_{n}\right|^{1 / n} \liminf _{n \rightarrow \infty: n \in N} \frac{s_{n}(f)}{R_{n}} \\
& =\tau_{R}(f) \liminf _{n \rightarrow \infty} \frac{s_{n}(f)}{R_{n}},
\end{aligned}
$$

which is the left side of (4.6). For the right side of (4.6), suppose $\tau_{R}(f)=1$, let $\alpha>1$ and define $f_{1}(z)=f(\alpha z)$. Then $\tau_{R}\left(f_{1}\right)=\alpha$ and Lemma 6 implies $\lim \inf _{n \rightarrow \infty} s_{n}\left(f_{1}\right) / R_{n}$ $\leqq P$. Since $s_{n}\left(f_{1}\right)=\alpha^{-1} s_{n}(f)$, we have $\lim \inf _{n \rightarrow \infty} s_{n}(f) / R_{n} \leqq P \alpha$. Letting $\alpha \rightarrow 1$, we obtain $\lim \inf _{n \rightarrow \infty} s_{n}(f) / R_{n} \leqq P$. Now suppose $\tau_{R}(f)=t$ and define $g(z)=f(z / t)$. Since $\tau_{R}(g)=1$, the previous inequality implies $\lim _{\inf }{ }_{n \rightarrow \infty} s_{n}(g) / R_{n} \leqq P$. But $s_{n}(g)$ $=t s_{n}(f)$ and therefore

$$
\tau_{R}(f) \liminf _{n \rightarrow \infty} \frac{s_{n}(f)}{R_{n}} \leqq P .
$$

This completes the proof of the theorem and establishes (1.5).

For the proof of (1.6), we require the following lemma.

LEMMA 8. If $0<\tau_{R}(f)<1$, then

$$
\limsup _{n \rightarrow \infty} \frac{r_{n}(f)}{R_{n}} \geqq 1 / P \text {. }
$$

Proof. Let $f(z)=\sum_{k=0}^{\infty} A_{k} z^{k}=\sum_{k=0}^{\infty}\left(a_{k} / R_{1} R_{2} \cdots R_{k}\right) z^{k}$. Since $\tau_{R}(f)=\limsup \left|a_{n}\right|^{1 / n}$ and $0<\tau_{R}(f)<1$, then there is an infinite set $N$ of positive integers such that $n \in N$ implies $\left|a_{n}\right|>\left|a_{k}\right|$ for $k>n$. Let $m$ be a positive integer, let $n \in N$ and suppose $k$ is an integer such that $0 \leqq k \leqq m-1$. The expression

$$
\frac{R_{n}^{k}\left(R_{1} \cdots R_{n}\right)}{a_{n}} \mathscr{S}^{n+k} f\left(R_{n} z\right)=\frac{a_{n+k} R_{n}^{k}}{a_{n} R_{n+1} \cdots R_{n+k}}+\frac{a_{n+k+1} R_{n}^{k+1}}{a_{n} R_{n+1} \cdots R_{n+k+1}} z+\cdots
$$


is the $k$ th normalized remainder of

$$
1+\frac{a_{n+1} R_{n}}{a_{n} R_{n+1}} z+\frac{a_{n+2} R_{n}^{2}}{a_{n} R_{n+1} R_{n+2}} z^{2}+\cdots
$$

By Lemma 4, there is an integer $k(n), 0 \leqq k(n) \leqq m-1$, such that

$$
\frac{a_{n+k(n)} R_{n}^{k(n)}}{a_{n} R_{n+1} \cdots R_{n+k(n)}}+\frac{a_{n+k(n)+1} R_{n}^{k(n)+1}}{a_{n} R_{n+1} \cdots R_{n+k(n)+1}} z+\cdots
$$

does not vanish in $|z| \leqq \mathscr{U}_{m}$. Therefore $\mathscr{S}^{n+k(n)} f\left(R_{n} z\right)$ has no zero in $|z| \leqq \mathscr{U}_{m}$, so that $r_{n+k(n)}(f) / R_{n} \geqq \mathscr{U}_{m}$ for all $n \in N$. It follows that $\left(r_{n+k(n)}(f) / R_{n+k(n)}\right)\left(R_{n+m-1} / R_{n}\right) \geqq \mathscr{U}_{m}$ and, therefore, $\lim \sup _{j \rightarrow \infty} r_{j}(f) / R_{j} \geqq \mathscr{U}_{m}$. By (3.1), $\lim \sup _{j \rightarrow \infty} r_{j}(f) / R_{j} \geqq 1 / P$, and this completes the proof.

The proof of (1.6) of Theorem $\mathrm{C}$ is contained in the following theorem.

THEOREM 2. If $\tau_{R}(f)>0$, then

$$
\tau_{R}(f) \limsup _{n \rightarrow \infty} \frac{r_{n}(f)}{R_{n}} \geqq 1 / P .
$$

Proof. Suppose first that $\tau_{R}(f)=1$, let $0<\alpha<1$, and define $f_{1}(z)=f(\alpha z)$. Then $r_{n}\left(f_{1}\right)=\alpha^{-1} r_{n}(f)$ and $\tau_{R}\left(f_{1}\right)=\alpha$. By Lemma 8 , $\lim _{\sup _{n \rightarrow \infty}} r_{n}\left(f_{1}\right) / R_{n} \geqq 1 / P$. Thus $\lim \sup _{n \rightarrow \infty} r_{n}(f) / R_{n} \geqq \alpha / P$ and, letting $\alpha \rightarrow 1$, we have $\lim \sup _{n \rightarrow \infty} r_{n}(f) / R_{n} \geqq 1 / P$.

Now suppose $\tau_{R}(f)=t$. If $t=\infty$, there is nothing to prove. For finite $t$, define $g(z)=f(z / t)$. Then $\tau_{R}(g)=1$ and $r_{n}(g)=t r_{n}(f)$. By the previous inequality, $\tau_{R}(f) \lim \sup _{n \rightarrow \infty} r_{n}(f) / R_{n} \geqq 1 / P$, which is the desired result.

5. Extremal functions. In this section, we construct extremal functions which show that $P$ is the sharp constant in each of the three inequalities of Theorem $\mathrm{C}$.

THEOREM 3. There is a function $f$ of $R$-type 1 such that $\lim _{\inf _{n \rightarrow \infty}} s_{n}(f) / R_{n}=P$.

Proof. Let $F(z)=\sum_{k=0}^{\infty} A_{k} z^{k}$ be the function constructed in Lemma 2. Recall that $c(F)=1, s_{n}(F) \geqq P,\left|A_{n}\right| \leqq 1$ and $\max \left\{\left|A_{n}\right|,\left|A_{n+1}\right|, \ldots,\left|A_{n+6}\right|\right\} \geqq 1 / 7000$ for all $n$. Let

and

$$
f(z)=\sum_{k=0}^{\infty}\left(A_{k} / R_{1} R_{2} \cdots R_{k}\right) z^{k} \quad\left(R_{0}=1\right)
$$

$$
x=\liminf _{n \rightarrow \infty} \frac{s_{n}(f)}{R_{n}} .
$$

Let $A$ denote an infinite set of positive integers such that $x=\lim _{n \rightarrow \infty: n \in A} s_{n}(f) / R_{n}$. For $n \in A$, define

and

$$
P_{n}(z)=z^{n} S_{n}\left(f ; R_{n} / z\right)\left(R_{1} R_{2} \cdots R_{n}\right) / R_{n}^{n}
$$

$$
Q_{n}(z)=z^{n} S_{n}(F ; 1 / z)=\sum_{k=0}^{n} A_{n-k} z^{k}
$$


The bound

$$
\left|P_{n}(z)-Q_{n}(z)\right| \leqq \sum_{k=1}^{n}|z|^{k}\left(1-\left(R_{n} R_{n-1} \cdots R_{n-k+1}\right) / R_{n}^{k}\right) \leqq(1-|z|)^{-1}
$$

holds for all $n \in A$ and $|z|<1$. Thus there is an infinite set of integers $B \subset A$ such that the sequence $\left\{P_{n}-Q_{n}\right\}_{n \in B}$ converges uniformly on compact subsets of $|z|<1$ to a function $g(z)=\sum_{k=0}^{\infty} \alpha_{k} z^{k}$ analytic in the unit disc. Since

$$
\alpha_{m}=\lim _{n \rightarrow \infty ; n \in B} A_{n-m}\left(1-\left(R_{n} R_{n-1} \cdots R_{n-m+1}\right) / R_{n}^{m}\right)=0,
$$

for $m=1,2,3, \ldots$, and $\alpha_{0}=0$, then $g \equiv 0$. For $n \in B$, we also have the bound $\left|Q_{n}(z)\right|<(1-|z|)^{-1},|z|<1$. Thus there is an infinite subset $C \subset B$ such that $\left\{Q_{n}\right\}_{n \in C}$ converges uniformly on compact subsets of $|z|<1$ to a function $Q(z)=\sum_{k=0}^{\infty} \beta_{k} z^{k}$ analytic in the unit disc. The bound $\max \left\{\left|\beta_{k}\right|,\left|\beta_{k+1}\right|, \ldots,\left|\beta_{k+6}\right|\right\}>1 / 7000$ holds for the coefficients of $Q$; in particular, $Q$ is not identically zero. The sequence $\left\{P_{n}(1 / z)\right\}_{n \in C}$ converges uniformly to $Q(1 / z)$ in $|z| \geqq 1 / \rho$ for all $\rho<1$. Moreover, $Q_{n}(1 / z)=\left(1 / z^{n}\right) S_{n}(F ; z)$ has a zero of modulus $P$ for all $n \in C$. Thus $Q(1 / z)$ has a zero of modulus $P$. If $\varepsilon>0$, it follows from Hurwitz's Theorem that $P_{n}(1 / z)$ has a zero of modulus at least $P-\varepsilon$ for $n \in C$ sufficiently large, i.e., if $\Gamma_{n}$ denotes the maximum modulus of the zeros of $P_{n}(1 / z)$, then $\Gamma_{n} \geqq P-\varepsilon$ for $n \in C$ sufficiently large. Since $\Gamma_{n}=R_{n}^{-1} s_{n}(f)$, then $s_{n}(f) / R_{n} \geqq P-\varepsilon$ for large $n \in C$. Therefore

$$
x=\lim _{n \rightarrow \infty ; n \in C} \frac{s_{n}(f)}{R_{n}} \geqq P-\varepsilon ;
$$

letting $\varepsilon \rightarrow 0$, we obtain the desired result.

THEOREM 4. There is a function $g$ of $R$-type 1 such that $\lim \sup r_{n}(g) / R_{n}=1 / P$.

Proof. Let $G(z)=\sum_{k=0}^{\infty} A_{k} z^{k}$ denote the function constructed in Lemma 3. We have $c(G)=1,\left|A_{n}\right| \leqq 1$ and $\max \left\{\left|A_{n}\right|,\left|A_{n+1}\right|, \ldots,\left|A_{n+6}\right|\right\} \geqq 1 / 7000$ for all $n$. Let

and

$$
g(z)=\sum_{k=0}^{\infty}\left(A_{k} / R_{1} R_{2} \cdots R_{k}\right) z^{k} \quad\left(R_{0}=1\right),
$$

$$
x=\limsup _{n \rightarrow \infty} \frac{r_{n}(g)}{R_{n}} .
$$

Let $A$ denote an infinite set of positive integers for which $x=\lim _{n \rightarrow \infty ; n \in A} r_{n}(g) / R_{n}$. For $m \in A$, define

$$
E_{m}(z)=\mathscr{S}^{m} G(z)-\left(R_{1} R_{2} \cdots R_{m} / R_{m}^{m}\right) \mathscr{S}^{m} g\left(R_{m} z\right)
$$

and let $0<\alpha<1$. If $m \in A,|z| \leqq \alpha$ and $N$ is a positive integer, then

$$
\begin{aligned}
\left|E_{m}(z)\right| & \leqq \sum_{k=1}^{\infty}\left|A_{m+k}\right|\left(1-R_{m}^{k} /\left(R_{m+1} \cdots R_{m+k}\right)\right)|z|^{k} \\
& \leqq \sum_{k=1}^{N}|z|^{k}\left(1-R_{m}^{k} /\left(R_{m+1} \cdots R_{m+k}\right)\right)+\sum_{k=N+1}^{\infty} \alpha^{k} \\
& \leqq\left(1-R_{m}^{N} /\left(R_{m+1} \cdots R_{m+N}\right)\right)(1-\alpha)^{-1}+\alpha^{N+1}(1-\alpha)^{-1} .
\end{aligned}
$$

Let $\varepsilon>0$ and choose $N$ so that $\alpha^{N+1}(1-\alpha)^{-1}<\varepsilon / 2$. Let $m_{0} \in A$ be a positive integer 
such that $m \geqq m_{0}$ implies $\left(1-R_{m}^{N} /\left(R_{m+1} \cdots R_{m+N}\right)\right)(1-\alpha)^{-1}<\varepsilon / 2$. Then the conditions $m \geqq m_{0}$ and $|z| \leqq \alpha$ imply $\left|E_{m}(z)\right|<\varepsilon$. Thus $\left\{E_{m}\right\}_{m \in A}$ converges uniformly to zero on compact subsets of $|z|<1$. For $m \in A$, we also have $\left|\mathscr{S}^{m} G(Z)\right| \leqq(1-|z|)^{-1}$. Thus there is an infinite subset $B \subset A$ of integers such that $\left\{\mathscr{S}^{m} G(Z)\right\}_{m \in B}$ converges uniformly on compact subsets of $|z|<1$ to a function $S(z)=\sum_{k=0}^{\infty} b_{k} z^{k}$. The relation

$$
\left|b_{k}\right|+\left|b_{k+1}\right|+\cdots+\left|b_{k+6}\right| \geqq 1 / 1000
$$

holds for all $k$; in particular $S \not \equiv 0$. Since $\mathscr{S}^{m} G(z)$ has a zero of modulus $1 / P$ for all $m \in B$, then $S(z)$ has a zero of modulus $1 / P$. Moreover, $S(z)$ is the uniform limit of the sequence $\left\{\left(R_{1} R_{2} \cdots R_{m} / R_{m}^{m}\right) \mathscr{S}^{m} g\left(R_{m} z\right)\right\}_{m \in B}$ and it follows from Hurwitz's Theorem that, if $\varepsilon>0$, then $\mathscr{S}^{\mathrm{m}} g\left(R_{m} z\right)$ has a zero of modulus at most $(1 / P)+\varepsilon$ for $m \in B$ sufficiently large. Therefore $r_{m}(g) / R_{m} \leqq(1 / P)+\varepsilon$ for large $m \in B$, and it follows that

$$
x=\lim _{m \rightarrow \infty: m \in B} \frac{r_{m}(g)}{R_{m}} \leqq(1 / P)+\varepsilon .
$$

Since $\varepsilon>0$ is arbitrary, we obtain $\lim \sup _{n \rightarrow \infty} r_{n}(g) / R_{n} \leqq 1 / P$ and this completes the proof.

For the left-hand side of $(1.5)$, we begin by considering the infinite matrix $\left(a_{m n}\right)$, where

$$
\begin{aligned}
a_{m n} & =2(m-n+1) / m^{2}, & & 1 \leqq n \leqq m, \\
& =0, & & m<n .
\end{aligned}
$$

It is easily verified that

(1) $\lim _{m \rightarrow \infty} a_{m n}=0, n=1,2,3, \ldots$,

(2) $\sup _{m} \sum_{n=1}^{\infty}\left|a_{m n}\right|=2$,

(3) $\lim _{m \rightarrow \infty} \sum_{n=1}^{\infty} a_{m n}=1$.

Thus $\left(a_{m n}\right)$ provides a regular method of summability. If $\left\{R_{n}\right\}_{n=1}^{\infty}$ is a nondecreasing sequence of positive numbers $\left(R_{0}=1\right)$ such that $R_{n+1} / R_{n} \rightarrow 1$, then $\left(a_{m n}\right)$ transforms the sequence $\left\{\log \left(R_{n} / R_{n-1}\right)\right\}_{n=1}^{\infty}$ into $\left\{2 \log \left(R_{1} R_{2} \cdots R_{n}\right)^{1 / n^{2}}\right\}_{n=1}^{\infty}$. Therefore

$$
\lim _{n \rightarrow \infty}\left[2 \log \left(R_{1} R_{2} \cdots R_{n}\right)^{1 / n^{2}}\right]=\lim _{n \rightarrow \infty}\left[\log \left(R_{n} / R_{n-1}\right)\right]=0
$$

or

$$
\lim _{n \rightarrow \infty}\left(R_{1} R_{2} \cdots R_{n}\right)^{1 / n^{2}}=1 .
$$

We use this result to prove the following lemma.

LEMMA 9. Let $\left\{R_{n}\right\}_{n=1}^{\infty}\left(R_{0}=1\right)$ be a nondecreasing sequence of positive numbers such that $\left(R_{1} R_{2} \cdots R_{n}\right)^{1 / n} \rightarrow \infty$ and $R_{n+1} / R_{n} \rightarrow 1$, as $n \rightarrow \infty$. For each pair of positive integers $m$ and $p$, let $x_{m p}$ be the largest root of the equation

$$
\frac{x^{m+p}}{R_{1} \cdots R_{m+p}}=\frac{x^{m}}{R_{1} \cdots R_{m}}+\frac{x^{m-1}}{R_{1} \cdots R_{m-1}}+\cdots+\frac{x}{R_{1}}+1 \text {. }
$$


Then

for $m=1,2,3, \ldots$

$$
\lim _{p \rightarrow \infty} \frac{x_{m p}}{\left(R_{1} \cdots R_{m+p}\right)^{1 /(m+p)}}=1
$$

Proof. For all $m$ and $p$ we have $x_{m p} \geqq\left(R_{1} \cdots R_{m+p}\right)^{1 /(m+p)}$, and therefore $x_{m p} \rightarrow \infty$ as $p \rightarrow \infty, m=1,2,3, \ldots$. Let $m$ be a positive integer and choose $p$ so large that $x_{m p}^{m} /\left(R_{1} \cdots R_{m}\right) \geqq x_{m p}^{m-k} /\left(R_{1} \cdots R_{m-k}\right)$, for $0 \leqq k \leqq m$. For such integers $p$ we have $x_{m p}^{m+p} /\left(R_{1} \cdots R_{m+p}\right) \leqq(m+1) x_{m p}^{m} /\left(R_{1} \cdots R_{m}\right)$ and hence

$$
x_{m p} \leqq(m+1)^{1 / p}\left(R_{m+1} \cdots R_{m+p}\right)^{1 / p} .
$$

Thus

$$
1 \leqq \frac{x_{m p}}{\left(R_{1} \cdots R_{m+p}\right)^{1 /(m+p)}} \leqq \frac{(m+1)^{1 / p}}{\left(R_{1} \cdots R_{m}\right)^{1 /(m+p)}}\left(R_{m+1} \cdots R_{m+p}\right)^{(1 / p)-(1 /(m+p))} .
$$

Since each of $(m+1)^{1 / p}$ and $\left(R_{1} \cdots R_{m}\right)^{1 /(m+p)}$ tends to 1 as $p \rightarrow \infty$, it is sufficient to show that

$$
\left(R_{m+1} \cdots R_{m+p}\right)^{(1 / p)-(1 /(m+p))}=\frac{\left(R_{1} \cdots R_{m+p}\right)^{m / p(m+p)}}{\left(R_{1} \cdots R_{m}\right)^{m / p(m+p)}} \rightarrow 1, \quad p \rightarrow \infty .
$$

Since $\left(R_{1} \cdots R_{m}\right)^{m / p(m+p)} \rightarrow 1$, it is sufficient to show that $\left(R_{1} \cdots R_{m+p}\right)^{1 / p(m+p)} \rightarrow 1$. Now

$$
\left(R_{1} \cdots R_{m+p}\right)^{1 / p(m+p)}=\left(R_{1} \cdots R_{m+p}\right)^{1 /(m+p)^{2}}\left[\left(R_{1} \cdots R_{m+p}\right)^{1 /(m+p)^{2}}\right]^{m / p},
$$

and we know that $\left(R_{1} \cdots R_{m+p}\right)^{1 /(m+p)^{2}} \rightarrow 1, p \rightarrow \infty$. Thus $\left(R_{1} \cdots R_{m+p}\right)^{1 / p(m+p)} \rightarrow 1$, and this completes the proof.

THEOREM 5. There is a function $\varphi$ of R-type 1 such that

$$
\liminf _{n \rightarrow \infty} \frac{\left(R_{1} R_{2} \cdots R_{n}\right)^{1 / n}}{R_{n}}=\liminf _{n \rightarrow \infty} \frac{s_{n}(\varphi)}{R_{n}} .
$$

Proof. Let $\left\{R_{n}\right\}_{n=1}^{\infty}\left(R_{0}=1\right)$ and $\left\{x_{m p}\right\}_{m, p=1}^{\infty}$ be defined as in Lemma 9. Let $\left\{n_{k}\right\}_{k=1}^{\infty}$ denote a sequence of positive integers such that

$$
\liminf _{n \rightarrow \infty} \frac{\left(R_{1} R_{2} \cdots R_{n}\right)^{1 / n}}{R_{n}}=\lim _{k \rightarrow \infty} \frac{\left(R_{1} \cdots R_{n_{k}}\right)^{1 / n_{k}}}{R_{n_{k}}}
$$

Let $m_{1}=n_{1}$, choose an integer $p_{1}$ such that $m_{1}+p_{1} \in\left\{n_{j}\right\}$ and

$$
\frac{x_{m_{1} p_{1}}}{\left(R_{1} \cdots R_{m_{1}+p_{1}}\right)^{1 /\left(m_{1}+p_{1}\right)}}<1+\frac{1}{2}
$$

and let $m_{2}=m_{1}+p_{1}$. If $m_{k}=m_{k-1}+p_{k-1} \in\left\{n_{j}\right\}$ has been chosen, choose the integer $p_{k}$ such that $m_{k}+p_{k} \in\left\{n_{j}\right\}$ and

$$
x_{m_{k} p_{k}} /\left(R_{1} \cdots R_{m_{k}+p_{k}}\right)^{1 /\left(m_{k}+p_{k}\right)}<1+1 /(k+1)
$$


and let $m_{k+1}=m_{k}+p_{k}$. Thus we inductively obtain the sequence $\left\{m_{j}\right\} \subset\left\{n_{j}\right\}$ such that (5.3) holds for $k=1,2,3, \ldots$ Now let

Note that

$$
\varphi(z)=1+z^{m_{1}} /\left(R_{1} \cdots R_{m_{1}}\right)+z^{m_{2}} /\left(R_{1} \cdots R_{m_{2}}\right)+\cdots .
$$

$$
\begin{aligned}
\left|S_{m_{j}}(\varphi ; z)\right| & \geqq \frac{|z|^{m_{j}}}{R_{1} \cdots R_{m_{j}}}-\frac{|z|^{m_{j-1}}}{R_{1} \cdots R_{m_{j-1}}}-\cdots-\frac{|z|^{m_{1}}}{R_{1} \cdots R_{m_{1}}}-1 \\
& >\frac{|z|^{m_{1}}}{R_{1} \cdots R_{m_{1}}}-\sum_{k=0}^{m_{j-1}} \frac{|z|^{k}}{R_{1} \cdots R_{k}}
\end{aligned}
$$

for $j=1,2,3, \ldots$ Moreover, if $x>x_{m p}$, then

$$
\frac{x^{m+p}}{R_{1} \cdots R_{m+p}}>\frac{x^{m}}{R_{1} \cdots R_{m}}+\cdots+\frac{x}{R_{1}}+1,
$$

since $x_{m p}$ is the largest positive root of (5.2). Thus if $|z|=x>x_{m_{j-1}, p_{j-1}}$, then $\left|S_{m_{j}}(\varphi ; z)\right|>0$. Therefore $s_{m_{j}}(\varphi) \leqq x_{m_{j-1} p_{j-1}}$. From (5.3) we have

$$
s_{m_{j}}(\varphi) /\left(R_{1} \cdots R_{m_{\jmath}}\right)^{1 / m_{\jmath}} \leqq 1+1 / j \text { for } j=1,2,3, \ldots
$$

Since $s_{n}(\varphi)=\infty$ for integers $n \notin\left\{m_{j}\right\}$, then

$$
\begin{aligned}
\liminf _{n \rightarrow \infty} \frac{s_{n}(\varphi)}{R_{n}} & =\liminf _{j \rightarrow \infty} \frac{s_{m_{j}}(\varphi)}{R_{m_{j}}} \\
& \leqq \liminf _{j \rightarrow \infty}\left[\frac{\left(R_{1} \cdots R_{m_{\jmath}}\right)^{1 / m_{j}}}{R_{m_{j}}}\left(1+\frac{1}{j}\right)\right] \\
& =\lim _{k \rightarrow \infty} \frac{\left(R_{1} \cdots R_{n_{k}}\right)^{1 / n_{k}}}{R_{n_{k}}}=\liminf _{n \rightarrow \infty} \frac{\left(R_{1} \cdots R_{n}\right)^{1 / n}}{R_{n}} .
\end{aligned}
$$

and this completes the proof.

\section{REFERENCES}

1. R. P. Boas, Jr. and R. C. Buck, Polynomial expansions of analytic functions, Ergebnisse der Mathematik und ihrer Grenzgebiete, Heft 19, Springer-Verlag, Berlin, 1958. MR 20 \#984.

2. J. D. Buckholtz, Zeros of partial sums of power series, Michigan Math. J. 15 (1968), 481-484. MR 38 \#3409.

3. —_ Zeros of partial sums of power series. II, Michigan Math. J. 17 (1970), 5-14. MR 41 \#3718.

4. J. D. Buckholtz and J. L. Frank, Whittaker constants, Proc. London Math. Soc. 3 (1971), 348-370.

5. M. B. Porter, On the polynomial convergents of a power series, Ann. of Math. (2) 8 (1906-1907), 189-192.

6. M. Tsuji, On the distribution of the zero points of sections of a power series. III, Japan. J. Math. 3 (1926), 49-51.

Department of Mathematics, University of Kentucky, Lexington, Kentucky 40506

Department of Mathematics, Virginia Polytechnic Institute and State University, BLACKSBURG, VIRGINIA 24061 